\title{
免疫克隆多目标优化算法求解约束优化问题"
}

尚荣华，焦李成，马文萍

(西安电子科技大学 智能信息处理研究所,陕西 西安 710071)

\section{Immune Clonal Multi-Objective Optimization Algorithm for Constrained Optimization}

\author{
SHANG Rong-Hua ${ }^{+}$, JIAO Li-Cheng, MA Wen-Ping \\ (Institute of Intelligent Information Processing, Xidian University, Xi’an 710071, China) \\ + Corresponding author: E-mail: rhshang@mail.xidian.edu.cn
}

\begin{abstract}
Shang RH, Jiao LC, Ma WP. Immune clonal multi-objective optimization algorithm for constrained optimization. Journal of Software, 2008,19(11):2943-2956. http://www.jos.org.cn/1000-9825/19/2943.htm
\end{abstract}

\begin{abstract}
In this paper, the disadvantages of some existing algorithms in handling constrained objective problems (COPs) are analyzed and an algorithm used for COPs-immune clonal multi-objective optimization algorithm (ICMOA) is proposed. This algorithm treats constrained optimization as a multi-objective optimization with two objectives. One objective is the original objective function and the other is obtained by the constraints. The concept of the Pareto-dominance in multi-objective optimization is introduced and each individual is implemented clone, mutation, selection and other operations based on the degree of its Pareto-dominance. The clone operation implements the searching for optimal solution in the global region and is available for getting a high quality solution. The mutation operation improves the searching for optimal solution in the local region and assures the diversity of the solutions. The selection operation guarantees the convergence to the optimal solution and improves the convergence speed. Based on the theorem of Markov chain, the global convergence of the new algorithm is proved. Compared with the existing algorithms, simulation results on 13 benchmark test problems show that the new algorithm has some advantages in convergence speed and precision.
\end{abstract}

Key words: clonal selection; multi-objective optimization; Pareto-dominance; constrained optimization; Markov chain

摘 要: 针对现有的约束处理技术的一些不足之处, 提出一种用于求解约束优化问题的算法一一免疫克隆多目标 优化算法(immune clonal multi-objective optimization algorithm, 简称 ICMOA). 算法的主要特点是通过将约束条件转 化为一个目标, 从而将问题转化为两个目标的多目标优化问题.引入多目标优化中的 Pareto-支配的概念, 每一个个体 根据其被支配的程度进行克隆、变异及选择等操作. 克隆操作实现了全局择优, 有利于得到高质量的解; 变异操作提 高算法的局部搜索能力,有利于所得解的多样性;选择操作有利于算法向着最优搜索, 而且加快了收敛速度.基于抗 体群的随机状态转移过程,证明该算法具有全局收玫性.通过对 13 个标准测试问题的测试,并与已有算法进行比较, 结果表明,该算法在收敛速度和求解精度上均具有一定的优势.

* Supported by the National Natural Science Foundation of China under Grant No.60703107 (国家自然科学基金); the National Basic Research Program of China under Grant No.2006CB705700 (国家重点基础研究发展计划(973)); the National High-Tech Research and Development Plan of China under Grant No.2006AA01Z107 (国家高技术研究发展计划(863))

Received 2007-01-03; Accepted 2007-08-24 
关键词: 克隆选择;多目标优化;Pareto-支配;约束优化;马尔可夫链 中图法分类号: TP18 文献标识码: A

人工免疫系统是模仿自然免疫系统功能的一种智能方法, 它实现一种受生物免疫系统启发, 学习外界物质 的自然防御机理的学习技术, 提供噪声忍耐、无教师学习、自组织、记忆等进化学习机理, 结合了分类器、神 经网络和机器推理等系统的一些优点, 因此具有提供新颖解决问题方法的潜力 ${ }^{[1]}$. 与进化算法相比, 克隆选择计 算已经表现出很多优异的特性, 如在提高收玫速度的同时, 较好地保持了种群的多样性, 从而能够比较有效地克 服诸如早熟收玫、骗问题等进化计算本身难以解决的问题 ${ }^{[2]}$.

文献[3]给出了 13 个相关的标准测试函数,数值实验结果表明,现有的求解约束优化问题的算法大都不能很好 地处理各种类型的约束优化问题,如等式约束、不等式约束、混合约束等.本文针对现有约束处理技术的一些不足 之处, 结合人工免疫系统中的免疫克隆算法, 提出了一种新的求解约束优化问题的算法. 该算法不使用惩罚函数, 也 不区分可行解与不可行解, 而是通过将约束条件转化为一个目标,将约束优化问题转化为两个目标的多目标优化问 题.引入人工免疫系统中的克隆、变异及选择等操作, 用于求解该类多目标优化问题. 克隆操作实现了全局择优, 有利于得到高质量的解;变异操作能够提高算法的局部搜索能力,有利于所得解的多样性;选择操作有利于算法向 着最优搜索而且加快了收敛速度.基于抗体群的随机状态转移过程,证明新算法具有全局收敛性.数值实验表明,新 算法在收敛速度和求解精度上优于已有的一些算法.

\section{1 问题的定义}

约束优化问题(constrained optimization problems, 简称 COPs)是工程应用领域经常遇到的一类数学规划问 题 ${ }^{[4]}$. 不失一般性,一个非线性规划问题(nonlinear programming, 简称 NLP)可表示为求目标函数 $f$ 的最小化 问题:

$$
\begin{cases}\text { minimize } & y=f(\boldsymbol{x}) \\ \text { subject to } & \boldsymbol{g}(\boldsymbol{x})=\left(g_{1}(\boldsymbol{x}), \ldots, g_{l}(\boldsymbol{x})\right) \leq 0 \\ & \boldsymbol{h}(\boldsymbol{x})=\left(h_{l+1}(\boldsymbol{x}), \ldots, h_{m}(\boldsymbol{x})\right)=0 \\ \text { where } & \boldsymbol{x}=\left(x_{1}, x_{2}, \ldots, x_{n}\right) \in \boldsymbol{X} \subseteq R^{n} \\ & \boldsymbol{X}=\left\{\left(x_{1}, x_{2}, \ldots, x_{n}\right) \mid l_{i} \leq x_{i} \leq u_{i}\right\} \\ & \boldsymbol{l}=\left(l_{1}, l_{2}, \ldots, l_{n}\right), \boldsymbol{u}=\left(u_{1}, u_{2}, \ldots, u_{n}\right)\end{cases}
$$

其中, $\boldsymbol{X}$ 为决策变量, $y$ 为目标函数, $\boldsymbol{X}$ 表示决策空间, $\boldsymbol{I}$ 和 $\boldsymbol{u}$ 分别为下界和上界.

定义 1 (可行域(feasible region)). 可行域 $X_{f}$ 是一个满足约束条件的决策变量的集合,定义如下:

$$
\boldsymbol{X}_{f}=\left\{\boldsymbol{x} \in \boldsymbol{X} \mid g_{i}(\boldsymbol{x}) \leq 0, i=1, \ldots, l ; h_{j}(\boldsymbol{x})=0, j=l+1, \ldots, m\right\}
$$

定义 2 (全局最优解 (global minimum)). $\boldsymbol{x}^{*} \in \boldsymbol{X}$ 称为 $f(x)$ 的全局最优解, 当且仅当 $\boldsymbol{x}^{*} \in \boldsymbol{X}_{\boldsymbol{f}}$, 且不存在 $\boldsymbol{x} \in \boldsymbol{X}_{\boldsymbol{f}}$, 使得 $f(x) \leq f\left(x^{*}\right)$.

\section{2 约束处理技术}

\section{1 已有的约束处理技术}

约束处理是工程优化中的一个关键部分. 现有的一些处理约束多目标优化的方法 ${ }^{[5,6]}$ 主要有保持可行解 法、罚函数法、约束锦标赛法、Ray-Tai-Seow 的约束处理方法. 惩罚函数法是处理约束条件的最常用方法, 其 本质是允许群体中的个体在一定程度上违反约束条件, 但必须对该个体依其违反约束条件的程度进行惩罚以 减小其被选择的概率. 个体违反约束条件的程度由惩罚函数来确定, 惩罚函数法虽然简单、易行, 但在实际操作 时, 罚因子的选取相当困难, 若罚因子太小, 则算法找到的最优解远离真正的最优解, 因此很难产生可行解; 若罚 
因子太大,则会引发计算上的困难,并且容易产生早熟收敛.

近年来,将约束优化问题转换为多目标优化问题(multi-objective optimization problems, 简称 MOPs)来处理 受到人们极大的重视. 文献[7]给出了几个基于进化多目标优化概念约束条件的处理技术. 这类算法的主要特点 是将约束条件作为一个或多个目标来看待. 基于上述思想的算法可分为两类:区分可行解与不可行解法和多目 标优化法.

区分可行解与不可行解法是将约束优化问题定义为具有两个目标的多目标优化问题.典型算法有:Deb 提 出的一种不需要任何罚参数的联赛选择算子, 并采用 3 个准则来比较成对的个体 ${ }^{[8]}$. Mezura 也采用了类似的思 想,提出一种简单的用于求解约束优化问题的多成员进化策略 ${ }^{[9]}$. Lin 等人认为在群体中保持一定比例的接近可 行域边界的不可行解,对于找到全局最优解很有帮助,并提出了一种新的个体比较准则 ${ }^{[10]}$.

多目标优化法的主要思想是将约束优化问题转换为多目标优化问题后,利用多目标优化技术处理. 比较典 型的有 Coello 等人相继提出的一种类似于向量评估遗传算法的群体多目标技术 ${ }^{[11]}$,一种基于 Pareto 排序过程 的方法 ${ }^{[12]}$ 等.

但是,上述方法大都是基于进化算法的,将免疫克隆多目标优化算法应用到求解约束优化问题的有关成果 还少有报道.

\section{2 本文的约束处理方法}

人工免疫系统中的克隆免疫算法较为成功地解决了多目标优化中解的多样性、均匀性以及很好的向着 Pareto-最优面的逼近性等问题 ${ }^{[13]}$. 本文借鉴了免疫克隆算法在解决多目标优化问题中的优势, 基于以前的工 作 ${ }^{[14,15]}$,结合约束优化问题的特点,提出了一种免疫克隆多目标优化处理约束优化问题的方法.

基于常用的构造惩罚函数方法对约束条件作如下处理,令

$$
\begin{aligned}
G_{j}(x)= \begin{cases}\max \left\{0, g_{j}(x)\right\}, & 1 \leq j \leq l \\
h_{j}(x) \mid, & l+1 \leq j \leq m\end{cases} \\
\\
G(x)=\sum_{j=1}^{m} G_{j}(x)
\end{aligned}
$$

则将约束条件转化为一个目标 $G(x)$.

$G(x)$ 与 $f(x)$ 构成两目标的矢量 $\boldsymbol{f}(\boldsymbol{x})$ :

$$
\boldsymbol{f}(\boldsymbol{x})=(f(\boldsymbol{x}), G(\boldsymbol{x}))
$$

于是,该由 $n$ 个决策变量、单个目标函数和 $l$ 个不等式约束和 $m-1$ 个等式约束条件组成的约束优化问题就 转化成 $n$ 个决策变量、两个目标函数的非约束多目标优化的问题了,如下所示:

$$
\left\{\begin{array}{l}
\text { minimize } \quad \boldsymbol{y}=\boldsymbol{f}(\boldsymbol{x})=(f(\boldsymbol{x}), G(\boldsymbol{x})) \\
\text { where } \quad \boldsymbol{x}=\left(x_{1}, x_{2}, \ldots, x_{n}\right) \in \boldsymbol{X} \\
\boldsymbol{X}=\left\{\left(x_{1}, x_{2}, \ldots, x_{n}\right) \mid l_{i} \leq x_{i} \leq u_{i}\right\} \\
\boldsymbol{I}=\left(l_{1}, l_{2}, \ldots, l_{n}\right), \boldsymbol{u}=\left(u_{1}, u_{2}, \ldots, u_{n}\right)
\end{array}\right.
$$

结合多目标优化和本文的约束处理方法,下面给出 4 个重要的定义.

定义 3(Pareto-支配(Pareto-dominance)). 称一个解 $\boldsymbol{x}^{*}$ 支配(Pareto 最优) 另一个解 $\boldsymbol{x}$ (记为 $\boldsymbol{x}^{*} \succ \boldsymbol{x}$ ) 当且仅当

$$
\forall i \in\{1, \ldots, p\}: f_{i}\left(\boldsymbol{x}^{*}\right) \leq f_{i}(\boldsymbol{x}) \wedge\left(\exists k \in\{1, \ldots, p\}: f_{i}\left(\boldsymbol{x}^{*}\right)<f_{i}(\boldsymbol{x})\right)
$$

定义 4(Pareto-最优解(Pareto-optimal)). 称解 $\boldsymbol{x}^{*}$ 为一个 Pareto-最优解(非支配解)当且仅当

$$
\neg \exists \boldsymbol{x} \in \boldsymbol{X}: \boldsymbol{x} \succ \boldsymbol{x}^{*}
$$

Pareto-最优解也称为非劣解或者有效解,所有的非支配解组成的矢量集称为非支配的.这些解除了它们都 在 Pareto-最优集里之外,没有明显的联系.

定义 5(Pareto-最优解集(Pareto-optimal set)). 在多目标优化过程中,所有 Pareto 最优解的集合 $P_{S}$ 定义 如下: 


$$
P_{S}=\left\{\boldsymbol{x}^{*} \mid \neg \exists \boldsymbol{x} \in \boldsymbol{X}_{f}: \boldsymbol{x} \succ \boldsymbol{x}^{*}\right\}
$$

定义 6(Pareto-优前沿面(Pareto-optimal front)). $P_{S}$ 中的解对应的目标函数值组成的集合 $P_{F}$ 称为 Pareto最优前沿面,定义如下:

$$
P_{F}=\left\{\boldsymbol{F}(\boldsymbol{x})=\left(f_{1}(\boldsymbol{x}), f_{2}(\boldsymbol{x}), \ldots, f_{p}(\boldsymbol{x})\right)^{T} \mid \boldsymbol{x} \in P_{S}\right\}
$$

将约束优化问题转换为多目标优化问题来处理的主要目的是为了避免使用惩罚函数. 虽然一个约束优化 问题可转换为一个双目标优化问题来处理, 但与一般的多目标优化问题仍然具有本质上的区别:对一般的多目 标优化问题而言, 其求解目的是为了找到一个分布均匀、多样性好, 而且能够较好地向着最优的 Pareto-前沿面 逼近的最优解集; 而由本文转换过来的 $f(x)$ 在可行域内又退化为一个单目标优化问题 $f(x)($ 此时, $G(x)=0)$, 这样, 其最优解仍然为一个点, 因而对 $f(x)$ 而言没有必要关心算法所得到的 Pareto-最优解的分布情况. 因此, 两者在寻 优过程上并不完全等价.

\section{3 求解约束优化问题的免疫克隆多目标优化算法}

\section{1 人工免疫系统的基本机理}

免疫是指机体对“自己(self)”和“非己(nonself)”的识别并排出非已的功能.具体来说,免疫是机体识别和排 除抗原性异物, 以维护自身生理平衡和稳定的功能.这种能够诱导机体免疫响应并与相应抗体发生特异性反应 的物质称为抗原. 在人工免疫系统中, 抗原一般指问题及其约束 ${ }^{[1]}$. 具体而言, 它是问题目标函数的函数, 是人工 免疫系统算法的始动因子以及重要的度量标准.抗体在免疫系统应答中起着主要的作用.免疫系统应答是针对 某种特定的“非已”物质即抗原而作出的特定的反应.每当检测到一个抗原时,抗体就会识别该抗原, 并作出是否 将该抗原通过克隆来增值的决定,这一过程称为克隆选择.

在本文算法中,抗体 $\boldsymbol{a}$ 对应于问题的候选解:

$$
\boldsymbol{a}=\left(a_{1}, a_{2}, \ldots, a_{n}\right)
$$

其中, $\boldsymbol{l}=\left(l_{1}, l_{2}, \ldots, l_{n}\right) \leq \boldsymbol{a}=\left(a_{1}, a_{2}, \ldots, a_{n}\right) \leq \boldsymbol{u}=\left(u_{1}, u_{2}, \ldots, u_{n}\right)$, 即 $l_{i} \leq a_{i} \leq u_{i}(i=1, \ldots, n)$. 因此,一个规模为 $m$ 的抗体群 可以表示为

$$
\boldsymbol{A}=\left\{\boldsymbol{a}_{1}, \boldsymbol{a}_{2}, \ldots, \boldsymbol{a}_{m}\right\}
$$

抗原对应于目标函数:

$$
\boldsymbol{y}=\boldsymbol{f}(\boldsymbol{a})=\left(f_{1}(\boldsymbol{a}), f_{2}(\boldsymbol{a})\right)
$$

其中, $f_{1}(\boldsymbol{a})=f(\boldsymbol{a}), f_{2}(\boldsymbol{a})=G(\boldsymbol{a})$.

\section{2 用于约束优化问题免疫克隆多目标优化算法}

克隆选择操作(clonal selection operation,简称 CSO) $)^{[1]}$ 是由亲和度诱导的抗体随机映射,实际上包括克隆操 作、免疫基因操作和克隆选择操作.对一个抗体群经过克隆选择操作以后, 将得到一个新的抗体群,其抗体群的 状态转移可以表示成如下的随机过程:

$$
A(\text { it }) \stackrel{\text { 克隆操作 } T_{c}^{C}}{\longrightarrow} A^{\prime}(\text { it }) \stackrel{\text { 免疫基因操作 } T_{\mathrm{g}}^{C}}{\longrightarrow} A^{\prime \prime}(\text { it }) \cup A^{\prime}(\text { it }) \stackrel{\text { 克隆选择操作 } T_{\mathrm{s}}^{C}}{\longrightarrow} A(i t+1)
$$

\subsection{1 克隆操作 $T_{c}^{C}$}

新算法中,在实施克隆操作之前,先通过 Pareto-支配的概念将抗体群 $A_{0}(i t)$ 中的抗体划分为支配抗体和非 支配抗体,即对于任意一个抗体 $\boldsymbol{a}^{*}(i t) \in \boldsymbol{A}_{0}(i t)$ ，如果不存在其他抗体 $\boldsymbol{a}(i t) \in \boldsymbol{A}_{0}(i t)$ 满足:

$$
\forall i \in\{1,2\}: f_{i}\left(\boldsymbol{a}^{*}(i t)\right) \geq f_{i}(\boldsymbol{a}(i t)) \wedge\left(\exists k \in\{1,2\}: f_{i}\left(\boldsymbol{a}^{*}(i t)\right)>f_{i}(\boldsymbol{a}(i t))\right)
$$

则称 $\boldsymbol{a}^{*}(i t)$ 为非支配抗体,否则称 $\boldsymbol{a}^{*}(i t)$ 为支配抗体.

抗体群 $\boldsymbol{A}_{0}(i t)$ 中的支配抗体组成支配抗体群 $\overline{\boldsymbol{A}}(i t)$, 非支配抗体组成非支配抗体群 $\boldsymbol{A}(i t)$. 对非支配抗体群 
$A($ it $)$ 执行克隆操作 $T_{c}^{C}$, 其定义为

$$
\boldsymbol{A}^{\prime}(i t)=T_{c}^{C}(\boldsymbol{A}(i t))=\left(T_{c}^{C}\left(\boldsymbol{a}_{1}(i t)\right), T_{c}^{C}\left(\boldsymbol{a}_{2}(i t)\right), \ldots, T_{c}^{C}\left(\boldsymbol{a}_{m}(i t)\right)\right)^{T}
$$

其中, $\boldsymbol{a}_{i}^{\prime}(i t)=T_{c}^{C}\left(\boldsymbol{a}_{\boldsymbol{i}}(i t)\right)=I_{i} \times \boldsymbol{a}_{\boldsymbol{i}}(i t), i=1,2, \ldots, m, I_{i}$ 是元素为 1 的 $q_{i}$ 维行向量,称为抗体 $\boldsymbol{a}_{\boldsymbol{i}}(i t)$ 的 $q_{i}$ 克隆.

$$
q_{i}(i t)=g\left(n_{c}, f\left(\boldsymbol{a}_{i}(i t)\right), \Theta_{i}\right)
$$

$\Theta_{i}$ 反映了抗体 $i$ 与其他抗体的亲合力.在新算法中,将其定义为

$$
\Theta_{i}=\min \left\{D_{i j}\right\}=\min \left\{\exp \left(\left\|\boldsymbol{a}_{i}-\boldsymbol{a}_{j}\right\|\right)\right\}, i \neq j ; i, j=1,2, \ldots, m
$$

其中, \|\|$\|$ 为欧氏距离, 在计算 $\Theta_{i}$ 时, 对 \|\|$\|$ 进行了归一化处理,即 $0 \leq\|\| \| \leq 1$. 显然,抗体亲合力越大, 相似程度越高, 从 而抗体间的抑制作用越强, 则 $\Theta_{i}$ 值越小.特别地, 当抗体亲合力为 0 时, $\Theta_{i}=1$. 进一步地, 记 $D=\left(D_{i j}\right)_{m \times m}$ $(i, j=1,2, \ldots, m)$ 为抗体-抗体亲合力矩阵,则 $D$ 是一个对称矩阵, 反映了种群的多样性.令

$$
q_{i}(k)=\operatorname{Int}\left(n_{c} \cdot \frac{f\left(\boldsymbol{a}_{i}(i t)\right)}{\sum_{j=1}^{m} f\left(\boldsymbol{a}_{j}(i t)\right)} \cdot \Theta_{i}\right), i=1,2, \ldots, m
$$

其中, $n_{c}>m$ 是与克隆规模有关的设定值,新算法中,令 $n_{c}=3 m ; \operatorname{Int}(\cdot)$ 表示取上整函数, $\operatorname{Int}(x)$ 表示大于 $x$ 的最小整 数. 因此,对单一抗体而言,其克隆规模是依据抗体-抗原亲合度、抗体-抗体亲合力自适应调整的,而且当受到抗 体间抑制小,而抗原刺激大时, 克隆规模也大, 反之就小. 克隆过后, 种群变为:

$$
\boldsymbol{A}^{\prime}(i t)=\left(\boldsymbol{a}_{1}^{\prime}(i t), \boldsymbol{a}_{2}^{\prime}(i t), \ldots, \boldsymbol{a}_{m}^{\prime}(i t)\right)^{T}
$$

其中,

$$
\boldsymbol{a}_{i}^{\prime}(i t)=\left\{\boldsymbol{a}_{i j}^{\prime}(i t)\right\}=\left\{\boldsymbol{a}_{i 1}(i t), \boldsymbol{a}_{i 2}(i t), \ldots, \boldsymbol{a}_{i q_{i}}(i t)\right\}, \quad \boldsymbol{a}_{i j}^{\prime}(i t)=\boldsymbol{a}_{i j}(i t)=\boldsymbol{a}_{i}(i t), j=1,2, \ldots, q_{i}
$$

所以,

$$
\boldsymbol{A}^{\prime}(i t)=\left(\boldsymbol{a}_{1}^{\prime}(i t), \boldsymbol{a}_{2}^{\prime}(i t), \ldots, \boldsymbol{a}_{N}^{\prime}(i t)\right)^{T}
$$

其中, $N=\sum_{i=1}^{m} q_{i}$.

\subsection{2 免疫基因操作 $T_{g}^{C}$}

免疫基因操作 $T_{g}^{C}$ 主要包括克隆重组操作 $T_{r}^{C}$ 和克隆变异操作 $T_{m}^{C}$. 克隆为多种重组策略共同作用提供了条 件,可以实现对同一抗体的不同克隆个体按照一定的概率采用不同的重组策略,如部分重组、整体重组等,从而 实现抗体间的协作,促进不同抗体间信息的交流,有利于增加种群多样性,提高算法的收敛速度.

\subsubsection{1 克隆重组操作 $T_{g}^{C}$}

本文采用多父体单形杂交算子,来实现克隆重组操作.多父体单形杂交算子使用 $n+1$ 个父体向量重组产生 后代,具体的操作步骤如下:

(1) 在 $R^{n}$ 中, $n+1$ 个独立的父体向量 $\boldsymbol{x}_{\boldsymbol{i}}(i=1,2, \ldots, n)$ 形成 $R^{n}$ 空间的一个单形( simplex).

(2) 计算出该 $n+1$ 个向量的中心 $\boldsymbol{o : o}=\frac{1}{n+1} \sum_{i=1}^{n+1} \boldsymbol{x}_{\boldsymbol{i}}$.

(3) 将单形沿各个方向 $\left(\boldsymbol{x}_{\boldsymbol{i}}-\boldsymbol{o}\right)$ 以一定的比例扩张.

(4) 从扩张后的单形中随机取一点即为一个后代.

例如,如图 1 所示,我们考虑二维空间中的 3 个向量, $\boldsymbol{x}^{(1)}, \boldsymbol{x}^{(2)}, \boldsymbol{x}^{(3)}$ 为 3 个向量,这 3 个向量形成一个单形,把 这个单形以比例 $(1+\varepsilon)$ 向外扩张 $\left(\varepsilon\right.$ 称为扩张比率), 令 $o=1 / 3\left(\boldsymbol{x}^{(1)}+\boldsymbol{x}^{(2)}+\boldsymbol{x}^{(3)}\right), \boldsymbol{y}^{(j)}=(1+\varepsilon)\left(\boldsymbol{x}^{(j)}-\boldsymbol{o}\right)(j=1,2,3)$, 由 $\boldsymbol{y}^{(1)}, \boldsymbol{y}^{(2)}, \boldsymbol{y}^{(3)}$ 产生一个新的单形, 在新单形中随机地取一点 $\boldsymbol{z}, \boldsymbol{z}=k_{1} \cdot \boldsymbol{y}^{(1)}+k_{2} \cdot \boldsymbol{y}^{(2)}+k_{3} \cdot \boldsymbol{y}^{(3)}$, 其中, $k_{1}, k_{2}, k_{3}$ 为 $[0,1]$ 中的 3 个随机数, 满足 $k_{1}+k_{2}+k_{3}=1, \mathbf{z}$ 即为一个三父代单形杂交算子产生的后代. 


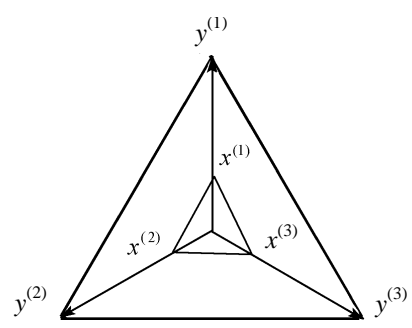

Fig.1 SPX with three parents in two-dimensional space

图 1 二维三父体单形杂交

由于多目标优化的解具有多样性的特点,在本算法的免疫克隆重组过程中,每 3 个父代抗体产生一个后代, 所以对于一个规模为 $N$ 的抗体群,一共产生的后代抗体的数目为 $\lfloor N / 3\rfloor$ 个. 对抗体群 $\boldsymbol{A}^{\prime}(i t)=\left(\boldsymbol{a}_{1}^{\prime}(i t), \boldsymbol{a}_{2}^{\prime}(i t), \ldots\right.$, $\left.\boldsymbol{a}_{N}^{\prime}(i t)\right)^{T}$ 的免疫克隆重组操作的主要计算步骤见算法 1.

算法 1. 免疫克隆重组算法.

\section{begin}

初始化 $j:=1$

while $(j \leq N-3)$ do

\{

求向量 $\left[\boldsymbol{A}^{\prime}(i t)\right](j,:),\left[\boldsymbol{A}^{\prime}(i t)\right](j+1,:),\left[\boldsymbol{A}^{\prime}(i t)\right](j+2,:)$ 的中心 $\boldsymbol{o}$ :

$$
\boldsymbol{o}=1 / 3\left(\left[\boldsymbol{A}^{\prime}(i t)\right](j,:)+\left[\boldsymbol{A}^{\prime}(i t)\right](j+1,:)+\left[\boldsymbol{A}^{\prime}(i t)\right](j+1,:)\right) .
$$

产生 $\boldsymbol{y}^{(1)}, \boldsymbol{y}^{(2)}, \boldsymbol{y}^{(3)}$ :

$$
\begin{aligned}
& \boldsymbol{y}^{(1)}=(1+\varepsilon)\left(\left[\boldsymbol{A}^{\prime}(i t)\right](j+1,:)-\boldsymbol{o}\right), \\
& \boldsymbol{y}^{(2)}=(1+\varepsilon)\left(\left[\boldsymbol{A}^{\prime}(i t)\right](j+1,:)-\boldsymbol{o}\right), \\
& \boldsymbol{y}^{(3)}=(1+\varepsilon)\left(\left[\boldsymbol{A}^{\prime}(i t)\right](j+2,:)-\boldsymbol{o}\right) ;
\end{aligned}
$$

随机产生两个 $[0,1]$ 之间的数: $\left[k_{1}, k_{2}\right]=\operatorname{rand}(1,2)$;

if $k_{1}+k_{2}>1$,

\{

$$
k_{1}=1 / 2 k_{1} ;
$$

$k_{2}=1 / 2 k_{2}$;

\}

else

$$
k_{3}=1-k_{1}-k_{2}
$$

end

由 $\boldsymbol{y}^{(1)}, \boldsymbol{y}^{(2)}, \boldsymbol{y}^{(3)}$ 产生一个新的单形: $\left[\boldsymbol{A}^{\prime \prime}(i t)\right]([j / 3\rceil,:)=k_{1} \cdot \boldsymbol{y}^{(1)}+k_{2} \cdot \boldsymbol{y}^{(2)}+k_{3} \cdot \boldsymbol{y}^{(3)}$;

$j=j+3$;

\}

end

\}

end

所以,经克隆重组操作 $T_{r}^{C}$ 后的抗体群变为

$$
\boldsymbol{A}^{\prime \prime}(i t)=T_{r}^{C}\left(\boldsymbol{A}^{\prime}(i t)\right)=\left(\boldsymbol{a}_{1}^{\prime \prime}(i t), \boldsymbol{a}_{2}^{\prime \prime}(i t), \ldots, \boldsymbol{a}_{p}^{\prime \prime}(i t)\right)^{T}, \quad p=\lfloor N / 3\rfloor
$$

为了更好地利用重组前的抗体群的信息, 令 


$$
\begin{aligned}
\boldsymbol{A}^{\prime \prime \prime}(i t) & =\boldsymbol{A}^{\prime \prime}(i t) \cup \boldsymbol{A}^{\prime}(i t) \\
& =\left(\boldsymbol{a}_{1}^{\prime \prime}(i t), \boldsymbol{a}_{2}^{\prime \prime}(i t), \ldots, \boldsymbol{a}_{p}^{\prime \prime}(i t), \boldsymbol{a}_{1}^{\prime}(i t), \boldsymbol{a}_{2}^{\prime}(i t), \ldots, \boldsymbol{a}_{N}^{\prime}(i t)\right)^{T} \\
& =\left(\boldsymbol{a}_{1}^{\prime \prime \prime}(i t), \boldsymbol{a}_{2}^{\prime \prime \prime}(i t), \ldots, \boldsymbol{a}_{p+N}^{\prime \prime \prime}(i t)\right)^{T}
\end{aligned}
$$

在重组和变异过程中,克隆后的抗体通过组合其他多个父代抗体的部分信息产生新的抗体,因此,新的抗体 继承了多个父代的部分基因.与遗传算法的交叉变异操作不同的是,新算法重组变异操作在搜索时,由于克隆算 子的作用,实现了在同一父代抗体周围的多个方向全局或局部搜索的同时进行,而不是只在单一方向的全局或 者局部搜索.

\subsubsection{2 克隆变异操作 $T_{m}^{C}$}

免疫学认为,亲合度成熟和抗体多样性的产生主要是依靠抗体的高频变异,而非交叉或重组.因此,与一般 遗传算法认为交叉是主要算子而变异是背景算子不同,在克隆选择算法中,更加强调变异的作用.

在新算法中,克隆变异操作采用的是自适应性变异 ${ }^{[16]}$,即在单一抗体周围产生一个变异的解的群体,利用局 部搜索增加了提高抗体与抗原亲和度的可能性(亲合力成熟).

自适应性变异将解的质量的好坏与搜索范围相关联,使适应值大的个体在较小范围内搜索而使适应值小 的个体在较大范围内搜索, 这样定义的变异算子将保护较好的解, 使搜索在其较小的领域内进行, 而对适应度不 好的解, 搜索的领域较大. 这使得变异能够根据解的质量自适应地调整搜索区域, 从而能够比较明显地提高搜索 的能力.其具体描述如下:

设 $\boldsymbol{s}=\left(v_{1}, v_{2}, \ldots, v_{n}\right)$ 是一个父解, $f(\boldsymbol{s})$ 是它的适应值, $f_{\max }$ 是所解问题的最大适应值, 定义其变异温度为

$$
T=1-\frac{f(s)}{f_{\max }}
$$

由于新算法是针对求解最小化的约束优化问题而设计的,是将单目标问题转化为多目标优化问题的方法. 所以,根据本文所要解决的问题的特点,将变异温度修改如下:

$$
T=1-\frac{f_{\min }}{f(s)}
$$

其中, $f_{\text {min }}$ 为所解问题的最小目标值, $f(s)$ 为个体 $\boldsymbol{s}$ 的目标函数值. 这样, 同样将解的质量的好坏与搜索范围相关联, 使接近于最优解的个体在较小范围内搜索而使远离最优解的个体在较大范围内搜索,从而保护了较好的解,使 搜索在其较小的领域内进行,而对离最优解较远的解,搜索的领域较大.

设分量 $v_{k} \in\left[a_{k}, b_{k}\right]$ 被选为进行变异,则变异后的解为

$$
\boldsymbol{s}^{\prime}=\left(v_{1}, \ldots, v_{k-1}, v_{k}^{\prime}, \ldots, v_{n}\right)
$$

其中,

$$
v_{k}^{\prime}= \begin{cases}v_{k}+\Delta\left(T, b_{k}-v_{k}\right), & \text { if } \operatorname{rnd}(2)=0 \\ v_{k}-\Delta\left(T, b_{k}-v_{k}\right), & \text { if } \operatorname{rnd}(2)=1\end{cases}
$$

其中, $r n d(2)$ 表示将随机、均匀地产生的正整数模 2 所得的结果, $T$ 为变异温度, $\Delta(T, y)$ 定义为

$$
\Delta(T, y)=y \cdot\left(1-r^{T^{\lambda}}\right)
$$

这里, $r$ 是 $[0,1]$ 上的一个随机数, $\lambda$ 是决定非一致性程度的一个参数, 它起着调整局部搜索区域的作用, 其取值一 般为 $2 \sim 5$.

具体地,该克隆变异操作是依概率 $p_{p+N}^{i}$ 对抗体群体 $A^{\prime \prime \prime}(i t)$ 进行变异操作,得到 $A^{\prime \prime \prime \prime}(i t)=T_{m}^{C}\left(A^{\prime \prime \prime}(i t)\right)$ 的主要 步骤见算法 2. 其中,对于不同的抗体所选取的 $k$ 值不一定相同, it 表示算法中当前的代数, $b u(i, k)$ 表示所选取的第 $i$ 个抗体的第 $k$ 个分量取值的上界, bu(i,k)表示所选取的第 $i$ 个抗体的第 $k$ 个分量取值的下界, 根据经验, 将 $\lambda$ 的值 取为 3 . 
算法 2. 免疫克隆变异算法.

\section{begin}

\{

初始化 $i:=1$

while $(i \leq p+N)$ do

\{

对抗体群 $\boldsymbol{A}^{\prime \prime \prime}(i t)$ 中的第 $i$ 个抗体随机选取第 $k$ 个分量 $\left[\boldsymbol{A}^{\prime \prime \prime}(i t)\right](i, k)$;

随机选取两个 $[0,1]$ 之间的数 $[a, r]=\operatorname{rand}(1,2)$;

if $a<0.5$

$$
\left[\boldsymbol{A}^{\prime \prime \prime}(i t)\right](i, k)=\left[\boldsymbol{A}^{\prime \prime \prime}(i t)\right](i, k)+\left(b u(i, k)-\left[\boldsymbol{A}^{\prime \prime \prime}(i t)\right](i, k)\right) \cdot\left(1-r^{\left(1-f_{\min } / f\left(\left[A^{\prime \prime}(i t)\right](i, i)\right)\right)^{\lambda}}\right)
$$

else

$$
\left[A^{\prime \prime \prime}(i t)\right](i, k)=\left[A^{\prime \prime \prime}(i t)\right](i, k)-\left(\left[A^{\prime \prime \prime}(i t)\right](i, k)-b d(i, k)\right) \cdot\left(1-r^{\left(1-f_{\min } / f\left(\left[A^{\prime \prime}(i t)\right](i, j)\right)\right)^{\lambda}}\right)
$$

end

$i=i+1$;

\}

end

\}

end

克隆变异操作完成后,得到新的抗体群:

$$
\boldsymbol{A}^{\prime \prime \prime \prime}(i t)=T_{m}^{C}\left(\boldsymbol{A}^{\prime \prime \prime}(i t)\right)=\left(T_{m}^{C}\left(\boldsymbol{a}_{1}^{\prime \prime \prime}(i t)\right), T_{m}^{C}\left(\boldsymbol{a}_{2}^{\prime \prime \prime}(i t)\right), \ldots, T_{m}^{C}\left(\boldsymbol{a}_{p+N}^{\prime \prime \prime}(i t)\right)\right)^{T}=\left(\boldsymbol{a}_{1}^{\prime \prime \prime}(i t), \boldsymbol{a}_{2}^{\prime \prime \prime}(i t), \ldots, \boldsymbol{a}_{p+N}^{\prime \prime \prime \prime}(i t)\right)^{T}
$$

\subsection{3 克隆选择操作 $T_{\mathrm{s}}^{C}$}

克隆选择操作 $T_{s}^{C}$ 是从抗体各自克隆增殖后的子代中选择优秀的个体, 从而形成新的种群, 是一个无性选 择过程.对抗体群 $\boldsymbol{A}^{\prime \prime \prime \prime}($ it $)$, 克隆选择操作 $T_{s}^{C}$ 表示如下:

$$
\begin{aligned}
\boldsymbol{A}^{\prime \prime \prime \prime}(i t) & =T_{s}^{C}\left(\boldsymbol{A}^{\prime \prime \prime \prime}(i t)\right) \\
& =T_{s}^{C}\left(\boldsymbol{a}_{1}^{\prime \prime \prime \prime}(i t), \boldsymbol{a}_{2}^{\prime \prime \prime}(i t), \ldots, \boldsymbol{a}_{p+N}^{\prime \prime \prime \prime}(i t)\right)^{T} \\
& =\left(\boldsymbol{a}_{1}^{\prime \prime \prime}(i t), \boldsymbol{a}_{2}^{\prime \prime \prime \prime}(i t), \ldots, \boldsymbol{a}_{N_{d o m}^{\prime \prime \prime \prime}}(i t)\right)^{T}
\end{aligned}
$$

在本文中,根据多目标优化中定义的 Pareto-最优概念将抗体群 $A^{\prime \prime \prime}(i t)$ 中的抗体分为两部分: 非支配抗体和 支配抗体. 克隆选择就是将抗体群 $\boldsymbol{A}^{\prime \prime \prime \prime}($ it $)$ 中的非支配抗体选择出来, 在式(31)中, $\boldsymbol{a}_{1}^{\prime \prime \prime \prime \prime}(i t), \boldsymbol{a}_{2}^{\prime \prime \prime \prime}(i t), \ldots, \boldsymbol{a}_{N_{d o m}}^{\prime \prime \prime \prime \prime}$ (it) 为 $\boldsymbol{A}^{\prime \prime \prime}(i t)$ 中的非支配抗体, $N_{\text {dom }}$ 为 $\boldsymbol{A}^{\prime \prime \prime}(i t)$ 中非支配抗体的个数.在新算法中,一般情况下, $N_{d o m}>m$, 此时,再进行二次 选择, 选出 $\boldsymbol{A}^{\prime \prime \prime \prime}(i t)$ 中违反约束程度较轻的 $m$ 个个体, 即

$$
\begin{aligned}
\boldsymbol{A}^{\prime \prime \prime \prime \prime}(i t) & =T_{s}^{C}\left(\boldsymbol{A}^{\prime \prime \prime \prime \prime}(i t)\right) \\
& =T_{\mathrm{s}}^{C}\left(\boldsymbol{a}_{1}^{\prime \prime \prime \prime}(i t), \boldsymbol{a}_{2}^{\prime \prime \prime \prime}(i t), \ldots, \boldsymbol{a}_{N_{\text {dom }}^{\prime \prime \prime \prime}}(i t)\right)^{T} \\
& =\left(\boldsymbol{a}_{1}^{\prime \prime \prime \prime \prime}(i t), \boldsymbol{a}_{2}^{\prime \prime \prime \prime \prime \prime}(i t), \ldots, \boldsymbol{a}_{m}^{\prime \prime \prime \prime \prime}(i t)\right)^{T}
\end{aligned}
$$

\section{3 求解约束优化问题的免疫克隆多目标优化算法}

由上述分析,结合免疫克隆操作、免疫基因操作以及克隆选择操作,本文给出了用于解决约束优化问题的 新的人工免疫系统算法——用于求解约束优化问题的免疫克隆多目标优化算法. 其主要步骤描述见算法 3. 其 中, $f^{*}$ 是目标函数 $f$ 的最优值, $f^{\text {best }}$ 是当前进化代数中的最好的目标函数值. 
算法 3. 求解约束优化问题的免疫克隆多目标优化算法.

\section{begin}

设定算法终止条件,给定抗体群规模 $m$; 初始化进化代数 it $:=0$;

随机产生初始抗体群 $\boldsymbol{A}(i t)=\left\{\boldsymbol{a}_{1}(i t), \boldsymbol{a}_{2}(i t), \ldots, \boldsymbol{a}_{m}(i t)\right\} \in I^{m}$;

while $\left(\left|f^{*}-f\right|>\varepsilon\right)$ do

\{

对 $\boldsymbol{A}(i t)$ 进行克隆操作 $T_{c}^{C}$, 得到: $\boldsymbol{A}^{\prime}(i t)=T_{c}^{C}(\boldsymbol{A}(i t))=\left(\boldsymbol{a}_{1}^{\prime}(i t), \boldsymbol{a}_{2}^{\prime}(i t), \ldots, \boldsymbol{a}_{m}^{\prime}(i t)\right)^{T}=\left(\boldsymbol{a}_{11}(i t), \boldsymbol{a}_{12}(i t), \ldots\right.$, $\left.\boldsymbol{a}_{1 q_{1}}(i t), \ldots, \boldsymbol{a}_{m 1}(i t), \boldsymbol{a}_{m 2}(i t), \ldots, \boldsymbol{a}_{m q_{m}}(i t)\right)^{T}=\left(\boldsymbol{a}_{1}^{\prime}(i t), \boldsymbol{a}_{2}^{\prime}(i t), \ldots, \boldsymbol{a}_{N}^{\prime}(i t)\right)^{T}, N=\sum_{i=1}^{m} q_{i} ;$

对 $\boldsymbol{A}^{\prime}(i t)$ 进行克隆重组操作 $T_{r}^{C}$, 得到: $\boldsymbol{A}^{\prime \prime}(i t)=T_{r}^{C}\left(\boldsymbol{A}^{\prime}(i t)\right)=\left(\boldsymbol{a}_{1}^{\prime \prime}(i t), \boldsymbol{a}_{2}^{\prime \prime}(i t), \ldots, \boldsymbol{a}_{p}^{\prime \prime}(i t)\right)^{T}, p=\lfloor N / 3\rfloor$;

令 $A^{\prime \prime \prime}(i t)=A^{\prime \prime}(i t) \cup A^{\prime}(i t)=\left(a_{1}^{\prime \prime \prime}(i t), \boldsymbol{a}_{2}^{\prime \prime \prime}(i t), \ldots, \boldsymbol{a}_{p+N}^{\prime \prime \prime}(i t)\right)^{T}$;

对 $\boldsymbol{A}^{\prime \prime \prime}($ it $)$ 进行克隆变异操作 $T_{m}^{C}$, 得到: $\boldsymbol{A}^{\prime \prime \prime \prime}(i t)=T_{m}^{C}\left(\boldsymbol{A}^{\prime \prime \prime}(i t)\right)=\left(T_{m}^{C}\left(\mathbf{a}_{1}^{\prime \prime \prime}(i t)\right), T_{m}^{C}\left(\mathbf{a}_{2}^{\prime \prime \prime}(i t)\right), \ldots\right.$, $\left.T_{m}^{C}\left(\boldsymbol{a}_{p+N}^{\prime \prime \prime}(i t)\right)\right)^{T}=\left(\boldsymbol{a}_{1}^{\prime \prime \prime \prime}(i t), \boldsymbol{a}_{2}^{\prime \prime \prime \prime}(i t), \ldots, \boldsymbol{a}_{p+N}^{\prime \prime \prime \prime}(i t)\right)^{T} ;$

对 $\boldsymbol{A}^{\prime \prime \prime \prime}($ it $)$ 进行克隆选择操作 $T_{s}^{C}$, 得到: $\boldsymbol{A}^{\prime \prime \prime \prime \prime}(i t)=T_{s}^{C}\left(\boldsymbol{A}^{\prime \prime \prime \prime}(i t)\right)=T_{s}^{C}\left(\boldsymbol{a}_{1}^{\prime \prime \prime}(i t), \boldsymbol{a}_{2}^{\prime \prime \prime \prime}(i t), \ldots, \boldsymbol{a}_{p+N}^{\prime \prime \prime \prime}(i t)\right)^{T}=$ $\left(\boldsymbol{a}_{1}^{\prime \prime \prime \prime}(\text { it }), \boldsymbol{a}_{2}^{\prime \prime \prime \prime}(\text { it }), \ldots, \boldsymbol{a}_{N_{\text {dom }}}^{\prime \prime \prime \prime \prime}(i t)\right)^{T}$;

if $N_{d o m}>m$

选出 $\boldsymbol{A}^{\prime \prime \prime \prime}(i t)$ 中违反约束程度较轻的 $m$ 个个体, 得到:

$$
\boldsymbol{A}^{\prime \prime \prime \prime \prime \prime}(i t)=T_{s}^{C}\left(\boldsymbol{A}^{\prime \prime \prime \prime \prime}(i t)\right)=\left(\boldsymbol{a}_{1}^{\prime \prime \prime \prime \prime}(i t), \boldsymbol{a}_{2}^{\prime \prime \prime \prime \prime}(i t), \ldots, \boldsymbol{a}_{m}^{\prime \prime \prime \prime \prime}(i t)\right)^{T} ;
$$

else

\{

选出 $\boldsymbol{A}^{\prime \prime \prime \prime \prime}($ it $)$ 中违反约束程度较轻的 $m-N_{\text {dom }}$ 个个体, 得到

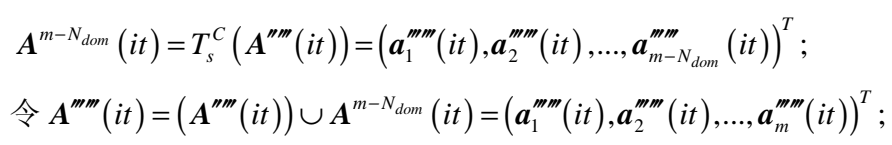

end

令 $\boldsymbol{A}(i t+1)=\boldsymbol{A}^{\prime \prime \prime \prime \prime \prime}(i t), \boldsymbol{a}_{i}(i t+1)=\boldsymbol{a}_{i}(i t)(i=1,2, \ldots, m)$, 则 $\boldsymbol{A}(i t+1)=\left\{\boldsymbol{a}_{1}(i t+1), \boldsymbol{a}_{2}(i t+1), \ldots, \boldsymbol{a}_{m}(i t+1)\right\} \in I^{m}$;

it $:=i t+1$;

\}

end

输出 $\boldsymbol{A}($ it $)$;

end

\section{4 算法分析}

\subsection{1 算法的收敛性分析}

定义 7(满意种群集). 称 $M=\left\{\boldsymbol{A} \mid \min (f(\boldsymbol{A}))=f^{*}, \forall \boldsymbol{A} \in I^{m}\right\}$ 为满意种群集, 则 $M$ 中的任意抗体群 $\boldsymbol{A}$ 中至少包 含 1 个最优解.

由于本算法采用实数编码,其数学模型可以描述为: 克隆选择过程是从一个状态到另一个状态的有记忆的 随机游动,因此可以用马尔可夫链(Markov chain)描述这一过程. 
在抗体种群空间 $S^{m}$ 上,抗体群从状态 $\boldsymbol{A}(i t)=\left(\boldsymbol{a}_{1}(i t), \boldsymbol{a}_{2}(i t), \ldots, \boldsymbol{a}_{m}(i t)\right)$ 经过克隆算子的作用后转移为状态 $\boldsymbol{A}(i t+1)=\left(\boldsymbol{a}_{1}(i t+1), \boldsymbol{a}_{2}(i t+1), \ldots, \boldsymbol{a}_{m}(i t+1)\right)$ 的过程为

$$
\boldsymbol{A}(i t+1)=T(\boldsymbol{A}(i t))=T_{s}^{C} \circ T_{r}^{C} \circ T_{m}^{C} \circ T_{c}^{C}(A(i t))
$$

记 $\boldsymbol{A}(i t)=X, \boldsymbol{A}(i t+1)=Y$, 则转移概率(transition probability):

(i) 当 $X \neq Y$ 时,

$$
p_{X Y}(i t)=p\{\boldsymbol{A}(i t+1)=Y \mid \boldsymbol{A}(i t)=X\}= \begin{cases}0, & i \in M, j \notin M \\ \prod_{j=1}^{m}\left(\sum_{i=1}^{q_{j}(i t)-1}\left(p_{m}^{i}\right)^{d(X, Y)}\left(1-p_{m}^{i}\right)^{l-d(X, Y)}\right), & \text { others }\end{cases}
$$

(ii) 当 $X=Y$ 时,

$$
p_{X Y}(i t)=p\{\boldsymbol{A}(i t+1)=Y \mid \boldsymbol{A}(i t)=X\}= \begin{cases}1-\sum_{L \neq Y}^{|M|} \prod_{j=1}^{m}\left(\sum_{i=1}^{q_{j}(i t)-1}\left(p_{m}^{i}\right)^{d(X, L)}\left(1-p_{m}^{i}\right)^{l-d(X, L)}\right), & i \in M, j \in M \\ 1-\sum_{L \neq Y}^{||^{m}|-| M \mid} \prod_{j=1}^{m}\left(\sum_{i=1}^{q_{j}(i t)-1}\left(p_{m}^{i}\right)^{d(X, L)}\left(1-p_{m}^{i}\right)^{l-d(X, L)}\right), & i \notin M, j \notin M\end{cases}
$$

其中, $L$ 代表克隆的中间状态, $p_{m}^{i}$ 为变异概率, $d(*)$ 代表欧式距离.

定理 1. 求解约束优化问题的免疫克隆多目标优化算法的抗体种群序列 $\{A(i t)=X, i t \geq 0\}$ 是有限非齐次可 约马尔可夫链.

证明:在给定精度后, 变量将在一定范围内取值,因此, 抗体种群 $\boldsymbol{A}(i t)=\left\{\boldsymbol{a}_{1}(i t), \boldsymbol{a}_{2}(i t), \ldots, \boldsymbol{a}_{m}(i t)\right\}$ 中的每个抗体 分量为离散实数, 个体的编码是有限的, 免疫克隆选择规划算法的状态变化都是在有限空间 $I^{m}$ 中进行的, 种群 是有限的. 由于 $q_{j}(i t)$ 与 it 时刻的种群状态有关, 所以 $p_{X Y}(i t)$ 与 it 有关, 因而是非齐次的.

由 $M$ 的定义可知, $M$ 是一个闭集,因为:

(1) 若 $X, Y \in M$, 则 $p_{X Y}(i t)>0, p_{Y X}(i t)>0$, 即 $X \leftrightarrow Y$;

(2) 若 $X \in M$ 且 $Y \notin M$, 则 $p_{X Y}(i t)=0$, 即 $X \nrightarrow Y$.

因此,除整个状态空间 $S^{m}$ 外,还有闭集 $M$, 所以 $\{A(i t)=X, i t \geq 0\}$ 是可约的.

综上所述,抗体种群序列 $\{\boldsymbol{A}(i t)=X, i t \geq 0\}$ 是有限非齐次可约马尔可夫链.

定理 2. 求解约束优化问题的免疫克隆多目标优化算法的抗体种群序列 $\{A(i t)=X, i t \geq 0\}$ 以概率 1 收玫到 满意种群集 $M$, 即对于任意的初始状态 $A_{0}$

$$
\lim _{i t \rightarrow \infty} P\left\{\boldsymbol{A}(i t) \in M \mid \boldsymbol{A}(0)=\boldsymbol{A}_{0}\right\}=1
$$

证明:不失一般性, 假设 $f(\boldsymbol{A})$ 只有唯一最小值解. 记 $F(\boldsymbol{A}(i t))=\min \left\{f\left(\boldsymbol{A}(i t)_{i}\right) ; i=1,2, \ldots, m\right\}$, 称

$$
P(i t)=p\left\{A(i t+1)=Y \mid \boldsymbol{A}(i t)=X ; X, Y \in I^{m}\right\}=\left(p_{X Y}(\boldsymbol{A}) ; X, Y \in I^{m}\right)
$$

为状态转移矩阵. 由于克隆算子中选择操作的性质,其选择概率为 $p_{s}^{i t}$ :

$$
p_{s}^{i t}= \begin{cases}0, & F(X)<F(Y) \\ 1, & F(X) \geq F(Y)\end{cases}
$$

则当 $F(Y) \leq F(X)$ 时有: $p_{X Y}(i t)=p\left\{T_{s}^{C} \circ T_{r}^{C} \circ T_{m}^{C} \circ T_{c}^{C}(X)=Y\right\}>0$;

当 $F(Y)>F(X)$ 时有: $p_{X Y}(i t)=0$,

记

$$
P(\infty)=\lim _{i t \rightarrow \infty} P(i t)=\left(P_{\infty}(X, Y) ; X, Y \in I^{m}\right)
$$

则

$$
P_{\infty}(X, Y) \begin{cases}>0, & F(X) \leq F(Y) \\ =0, & F(X)>F(Y)\end{cases}
$$


显然, $P(\infty)$ 是随机矩阵. 由于 $M$ 是 $P(\infty)$ 的非周期正常返类, 而 $\bar{M}=S^{m}-M$ 是非常返类, 因此 $\{A(i t), i t \geq 0\}$ 是强遍历的;对于任意初始状态 $A_{0}$, 有

$$
\lim _{i t \rightarrow \infty} P\left\{A(i t) \in Y \mid A(0)=A_{0}\right\}=\pi_{\infty}(Y)
$$

且 $\sum_{Y \in M} \pi_{\infty}(Y)=1$.于是,

$$
\lim _{i t \rightarrow \infty} P\left\{\boldsymbol{A}(i t) \in M \mid A(0)=A_{0}\right\}=\sum_{Y \in M} \pi_{\infty}(Y)=1
$$

\subsection{2 算法的复杂度分析}

该算法,每一代中的时间复杂度主要由克隆操作、重组操作、变异操作和克隆选择 4 部分组成. 设 $m$ 为抗 体群的规模, $N$ 为克隆之后的种群规模, $N+p=N+\lfloor N / 3\rfloor$ 为重组操作之后的种群规模, $n_{c}>m$ 是与克隆规模有关 的设定值, 根据经验, 本文设置为 $n_{c}=3 m$, 则在一代运行中, 克隆操作的时间复杂度最差为 $O\left(n_{c}\right)$; 重组操作的时间 复杂度为 $O(N)$; 变异操作的时间复杂度为 $O(p+N)$; 克隆选择操作的时间复杂度为 $O\left(N+\left(m n_{c}\right)^{2}+N_{\text {dom }}\right)$. 所以, 在 一代的运行中,算法的时间复杂度最差为

$$
\begin{aligned}
O\left(m n_{c}\right)+O(N)+O(p+N)+O\left(N+\left(n_{c}\right)^{2}+N_{\text {dom }}\right) & =O\left(m n_{c}+N+(p+N)+N+\left(n_{c}\right)^{2}+N_{\text {dom }}\right) \\
& =O\left(m n_{c}+3 N+\lfloor N / 3\rfloor+\left(n_{c}\right)^{2}+N_{\text {dom }}\right) \\
& =O\left(m \cdot 3 m+3 N+\lfloor N / 3\rfloor+(3 m)^{2}+N_{\text {dom }}\right) \\
& =O\left(10 m^{2}+3 N+\lfloor N / 3\rfloor+N_{\text {dom }}\right)
\end{aligned}
$$

又因为 $N_{d o m}$ 和 $N$ 与 $m$ 同阶,所以在一代的运行中,算法的时间复杂度最差为

$$
O\left(10 m^{2}+3 N+\lfloor N / 3\rfloor+N_{\text {dom }}\right)=O\left(m^{2}\right)
$$

\section{4 仿真实验及其结果比较}

\section{1 测试问题}

本文使用了 13 个标准的测试函数来检验新算法. 这些测试问题都是高维的带约束条件的函数优化问题,约 束条件有等式约束、不等式约束以及两者的混合 ${ }^{[3]}$,具体的可参见文献[3].

\section{2 测试结果及性能分析}

为了验证新算法的有效性,将本文用于求解约束优化问题的免疫克隆多目标优化算法(ICMOA)与文献[5] 中的同态映射法(KM)、文献[3]所述的基于随机排序的约束进化策略(RY)、文献[6]所述的用于约束优化问题 (self-adaptive fitness formulation, 简称 SAFF)的适应值方法, 以及文献[9]所述的一种用于约束优化问题(simple multimembered evolution strategy,简称 SMES)的简单的多成员进化算法在解决上述约束问题时的性能进行了 比较.

根据经验, 算法 ICMOA 的运行参数如下: 抗体群规模 $m=100$, 克隆比例 $q=3$, 个体变异概率 $p_{m}=1, \lambda=3$, 扩张比 率 $\varepsilon=4$. 算法 KM,RY,SAFF 和 SMES 的运行参数均按照相应文献中的最优参数设置. 在上述情况下, 针对一个测 试问题,各种算法的函数评价次数见表 1 .

Table 1 Function evaluation numbers of five algorithms

表 15 种算法的函数评价次数

\begin{tabular}{c|ccccc}
\hline Algorithms & KM & RY & SAFF & SMES & ICMOA \\
Function evaluation numbers & 1400000 & 350000 & 1400000 & 240000 & 350000 \\
\hline
\end{tabular}

由表 1 可以看出, KM 算法和 SAFF 算法的适应值函数评价次数最大,RY 算法和 ICMOA 算法的适应值函 数评价次数居中, SMES 算法的适应值函数评价次数最小, 从而说明 $\mathrm{KM}$ 算法和 SAFF 算法的计算代价最大, RY 算法和 ICMOA 算法的计算代价较小,SMES 算法的计算代价最小. 
表 2 为 5 种算法对这些标准的测试函数的实验结果. 其中,对每个测试问题在相同条件下独立运行 30 次, 记录其最好结果、最差结果和平均结果.表 2 中,“一”表示没有相应的实验数据.

Table 2 Simulation results of KM, RY, SAFF, SMES and ICMOA on COPs

表 2 KM,RY,SAFF,SMES 和 ICMOA 五种算法关于 COPs 的仿真结果

\begin{tabular}{|c|c|c|c|c|c|c|c|}
\hline Problem & Optimal & Results & KM & RY & SAFF & SMES & ICMOA \\
\hline g01 & -15.000 & $\begin{array}{c}\text { best } \\
\text { mean } \\
\text { worst } \\
\text { st.dev }\end{array}$ & $\begin{array}{c}-14.7864 \\
-14.7082 \\
-14.6154 \\
3.1 \mathrm{E}-02\end{array}$ & $\begin{array}{c}-15.000 \\
-15.000 \\
-15.000 \\
0\end{array}$ & $\begin{array}{c}-15.000 \\
-15.000 \\
-15.000 \\
0\end{array}$ & $\begin{array}{c}-15.000 \\
-15.000 \\
-15.000 \\
0\end{array}$ & $\begin{array}{c}-15.000 \\
-15.000 \\
-15.000 \\
0\end{array}$ \\
\hline g02 & -0.803619 & $\begin{array}{c}\text { best } \\
\text { mean } \\
\text { worst } \\
\text { st.dev }\end{array}$ & $\begin{array}{c}-0.79953 \\
-0.79671 \\
-0.79119 \\
7.0 \mathrm{E}-03 \\
\end{array}$ & $\begin{array}{c}-0.803515 \\
-0.782134 \\
-0.721254 \\
3.7 \mathrm{E}-02 \\
\end{array}$ & $\begin{array}{c}-0.80297 \\
-0.79121 \\
-0.72157 \\
1.9 \mathrm{E}-02 \\
\end{array}$ & $\begin{array}{c}-0.803601 \\
-0.784257 \\
-0.750984 \\
3.8 \mathrm{E}-02 \\
\end{array}$ & $\begin{array}{c}-0.803619 \\
-0.789512 \\
-0.697845 \\
6.5 \mathrm{E}-02 \\
\end{array}$ \\
\hline g03 & -1.000 & $\begin{array}{l}\text { best } \\
\text { mean } \\
\text { worst } \\
\text { st.dev } \\
\end{array}$ & $\begin{array}{l}-0.9997 \\
-0.9989 \\
-0.9978 \\
9.5 E-04 \\
\end{array}$ & $\begin{array}{c}-1.000 \\
-1.000 \\
-1.000 \\
0 \\
\end{array}$ & $\begin{array}{c}-1.000 \\
-1.000 \\
-1.000 \\
0 \\
\end{array}$ & $\begin{array}{c}-1.000 \\
-1.000 \\
-0.999 \\
3.7 \mathrm{E}-05 \\
\end{array}$ & $\begin{array}{c}-1.000 \\
-1.000 \\
-1.000 \\
0 \\
\end{array}$ \\
\hline g04 & -30665.539 & $\begin{array}{c}\text { best } \\
\text { mean } \\
\text { worst } \\
\text { st.dev }\end{array}$ & $\begin{array}{c}-30664.5 \\
-30655.3 \\
-30645.9 \\
1.4 \mathrm{E}+00\end{array}$ & $\begin{array}{c}-30665.539 \\
-30665.539 \\
-30665.539 \\
0\end{array}$ & $\begin{array}{c}-30665.50 \\
-30664.20 \\
-30663.30 \\
2.3 \mathrm{E}-01\end{array}$ & $\begin{array}{c}-30665.539 \\
-30665.539 \\
-30665.539 \\
0\end{array}$ & $\begin{array}{c}-30665.539 \\
-30665.539 \\
-30665.539 \\
0\end{array}$ \\
\hline g05 & 5126.4981 & $\begin{array}{c}\text { best } \\
\text { mean } \\
\text { worst } \\
\text { st.dev }\end{array}$ & - & $\begin{array}{c}5126.497 \\
5129.126 \\
5146.254 \\
5.3 \mathrm{E}+00 \\
\end{array}$ & $\begin{array}{c}5126.989 \\
5431.884 \\
6081.547 \\
4.0 \mathrm{E}+03 \\
\end{array}$ & $\begin{array}{c}5126.610 \\
5237.693 \\
5302.656 \\
4.9 \mathrm{E}+01 \\
\end{array}$ & 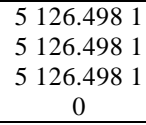 \\
\hline g06 & -6961.814 & $\begin{array}{c}\text { best } \\
\text { mean } \\
\text { worst } \\
\text { st.dev }\end{array}$ & $\begin{array}{c}-6952.141 \\
-6342.667 \\
-5473.982 \\
7.4 \mathrm{E}+02\end{array}$ & $\begin{array}{c}-6961.814 \\
-6874.457 \\
-6352.867 \\
8.9 \mathrm{E}+01\end{array}$ & $\begin{array}{c}-6961.800 \\
-6961.800 \\
-6961.800 \\
0\end{array}$ & $\begin{array}{c}-6961.814 \\
-6961.284 \\
-6960.482 \\
5.3 \mathrm{E}-01\end{array}$ & $\begin{array}{l}-6961.814 \\
-6961.187 \\
-6960.945 \\
3.8 \mathrm{E}-03\end{array}$ \\
\hline g07 & 24.306 & $\begin{array}{l}\text { best } \\
\text { mean } \\
\text { worst } \\
\text { st.dev }\end{array}$ & - & $\begin{array}{c}24.307 \\
24.371 \\
24.638 \\
2.8 \mathrm{E}-02\end{array}$ & $\begin{array}{c}24.48 \\
26.53 \\
28.46 \\
7.3 \mathrm{E}-01\end{array}$ & $\begin{array}{c}24.327 \\
24.471 \\
24.833 \\
6.5 \mathrm{E}-01\end{array}$ & $\begin{array}{c}24.306 \\
24.311 \\
24.324 \\
1.3 \mathrm{E}-01\end{array}$ \\
\hline g08 & -0.095825 & $\begin{array}{c}\text { best } \\
\text { mean } \\
\text { worst } \\
\text { st.dev } \\
\end{array}$ & $\begin{array}{c}-0.095825 \\
-0.089156 \\
-0.029143 \\
4.5 \mathrm{E}-01 \\
\end{array}$ & $\begin{array}{c}-0.095825 \\
-0.095825 \\
-0.095825 \\
5.3 \mathrm{E}-11 \\
\end{array}$ & $\begin{array}{c}-0.095825 \\
-0.095825 \\
-0.095825 \\
3.7 \mathrm{E}-08 \\
\end{array}$ & $\begin{array}{c}-0.095825 \\
-0.095825 \\
-0.095825 \\
0 \\
\end{array}$ & $\begin{array}{c}-0.095825 \\
-0.095825 \\
-0.095825 \\
0 \\
\end{array}$ \\
\hline g09 & 680.630 & $\begin{array}{c}\text { best } \\
\text { mean } \\
\text { worst } \\
\text { st.dev }\end{array}$ & $\begin{array}{c}680.91 \\
681.16 \\
683.18 \\
3.1 \mathrm{E}-01 \\
\end{array}$ & $\begin{array}{l}680.630 \\
680.656 \\
680.763 \\
2.1 \mathrm{E}-02 \\
\end{array}$ & $\begin{array}{c}680.64 \\
680.72 \\
680.87 \\
4.9 \mathrm{E}-02 \\
\end{array}$ & $\begin{array}{l}680.632 \\
680.643 \\
680.719 \\
1.7 \mathrm{E}-02 \\
\end{array}$ & $\begin{array}{l}680.630 \\
680.632 \\
680.671 \\
8.3 \mathrm{E}-03\end{array}$ \\
\hline g10 & 7049.25 & $\begin{array}{l}\text { best } \\
\text { mean } \\
\text { worst } \\
\text { st.dev }\end{array}$ & - & $\begin{array}{c}7054.316 \\
7559.192 \\
8835.655 \\
4.5 E+02\end{array}$ & $\begin{array}{l}7061.34 \\
7627.89 \\
8288.79 \\
3.9 E+02\end{array}$ & $\begin{array}{c}7051.903 \\
7253.047 \\
7638.366 \\
9.2 E+01\end{array}$ & $\begin{array}{cc}7 & 049.284 \\
7 & 049.289 \\
7 & 049.291 \\
1.7 & \mathrm{E}-01\end{array}$ \\
\hline g11 & 0.750 & $\begin{array}{c}\text { best } \\
\text { mean } \\
\text { worst } \\
\text { st.dev } \\
\end{array}$ & $\begin{array}{c}0.750 \\
0.750 \\
0.750 \\
3.4 \mathrm{E}-05 \\
\end{array}$ & $\begin{array}{c}0.750 \\
0.750 \\
0.750 \\
2.5 \mathrm{E}-08 \\
\end{array}$ & $\begin{array}{c}0.750 \\
0.750 \\
0.750 \\
5.2 \mathrm{E}-07 \\
\end{array}$ & $\begin{array}{c}0.750 \\
0.750 \\
0.750 \\
2.1 \mathrm{E}-15 \\
\end{array}$ & $\begin{array}{c}0.750 \\
0.750 \\
0.750 \\
0 \\
\end{array}$ \\
\hline g12 & -1.000000 & $\begin{array}{l}\text { best } \\
\text { mean } \\
\text { worst } \\
\text { st.dev }\end{array}$ & $\begin{array}{c}-0.999999857 \\
-0.999134613 \\
-0.991950498 \\
2.6 \mathrm{E}-01\end{array}$ & $\begin{array}{c}-1.000000 \\
-1.000000 \\
-1.000000 \\
0\end{array}$ & $\begin{array}{c}-1.000000 \\
-1.000000 \\
-1.000000 \\
0\end{array}$ & $\begin{array}{c}-1.000000 \\
-1.000000 \\
-1.000000 \\
0\end{array}$ & $\begin{array}{c}-1.000000 \\
-1.000000 \\
-1.000000 \\
0\end{array}$ \\
\hline g13 & 0.053950 & $\begin{array}{l}\text { best } \\
\text { mean } \\
\text { worst } \\
\text { st.dev }\end{array}$ & $\begin{array}{c}0.054 \\
0.164 \\
0.557 \\
1.1 \mathrm{E}-01 \\
\end{array}$ & $\begin{array}{c}0.053957 \\
0.157006 \\
0.216915 \\
1.7 \mathrm{E}-01\end{array}$ & - & $\begin{array}{c}0.053986 \\
0.166385 \\
0.468294 \\
3.1 \mathrm{E}-01\end{array}$ & $\begin{array}{c}0.053950 \\
0.054647 \\
0.056872 \\
1.3 \mathrm{E}-03\end{array}$ \\
\hline
\end{tabular}

从表 2 中可以看出,RY,SAFF,SMES 与 ICMOA 求得的最优结果明显优于 KM 求得的最优结果. 对测试问题 $\mathrm{g} 01, \mathrm{~g} 03, \mathrm{~g} 04, \mathrm{~g} 08, \mathrm{~g} 11$ 和 g12,RY,SAFF,SMES 与 ICMOA 四种算法每一次运行中都能找到最优结果,而对其他 7 个测试问题,ICMOA 算法运行的结果在最好结果、最差结果和平均结果诸方面都要好于 RY,SAFF 和 SMES 这 
3 种算法得到的结果. 在这 13 个测试问题中,对于测试问题 g10, 虽然新算法没有找到最优的结果,但是也得到一 个比较接近于最优解的结果 7049.284, 该结果明显要好于其他 4 种算法得到的结果. 该最优结果在最优点 $\boldsymbol{x}^{*}=$ (579.279329842548,1360.00457578913,5109.99998325897,182.014807943464,295.601133997103,217.983712354687, 286.413655678014,395.601039960173). 从而说明新算法具有很强的有效性和鲁棒性.

\section{5 结 论}

基于人工免疫系统中的免疫优势概念和抗体克隆选择学说以及多目标优化的方法,本文提出一种用于解 决约束优化问题的新算法——免疫克隆多目标优化算法用于约束多目标优化问题(ICMOA). 新算法的主要特 点是通过将约束条件转化为一个目标, 从而将约束优化问题转化为两个目标的多目标优化问题.引入了多目标 优化中的 Pareto-支配的概念,每一个个体根据其被支配的程度进行克隆、变异及选择等操作.算法克服了传统 的约束条件处理方法所引发的一些问题. 文中对算法的收玫性进行了分析和证明.最后对 13 个标准的测试函数 进行了测试,测试结果与理论分析一致, 表明新算法具有很强的处理各种类型约束优化问题的能力,即具有较好 的收玫性、较少的计算代价、很强的全局搜索能力,从而说明新算法具有很强的有效性和鲁棒性. 虽然新算法 对于求解这些测试函数取得了很好的效果, 但是如何更好地将其应用到解决实际问题中去, 怎样才能更好地以 一些实际问题来检验新算法的效果,将是我们进一步要做的工作.

\section{References:}

[1] Jiao LC, Du HF, Liu F, Gong MG. Immunological Computation for Optimization, Learning and Recognition. Beijing: Science Press, 2006 (in Chinese).

[2] Jiao LC, Wang L. A novel genetic algorithm based on immunity. IEEE Trans. on Systems, Man, and Cybernetics-Part A: Systems and Humans, 2000,30(5):552-561.

[3] Runarsson TP, Yao X. Stochastic ranking for constrained evolutionary optimization. IEEE Trans. on Evolutionary Computation, 2000,4(3):284-294.

[4] Zhou YR, Li YX, Wang Y, Kang LS. A pareto strength evolutionary algorithm for constrained optimization. Journal of Software, 2003,14(7):1243-1249 (in Chinese with English abstract). http://www.jos.org.cn/1000-9825/14/1243.htm

[5] Michalewicz Z, Deb K, Schmidt M, Stidsen T. Test-Case generator for nonlinear continuous parameter optimization techniques. IEEE Trans. on Evolutionary Computation, 2000,4(3):197-215.

[6] Farmani R, Wright JA. Self-Adaptive fitness formulation for constrained optimization. IEEE Trans. on Evolutionary Computation, 2003,7(5):445-455.

[7] Mezura-Montes E, Coello CAC. A survey of constraint-handling techniques based on evolutionary multiobjective optimization. Technical Report, EVOCINV-04-2006, Departamento de Computación, Evolutionary Computation Group at CINVESTAV, 2006. http://delta.cs.cinvestav.mx/ ccoello/2006.html

[8] Deb K. An efficient constraint handling method for genetic algorithms. Computational Methods for Applied Mechanical Engineering, 2000,186(2-4):311-338.

[9] Mezura-Montes E, Coello Coello AC. A simple multimembered evolution strategy to solve constrained optimization problems. IEEE Trans. on Evolutionary Computation, 2005,9(1):1-17.

[10] Lin D, Li MQ, Kou JS. A GA-based method for solving con strained optimization problems. Journal of Software, 2001,12(4): 628-632 (in Chinese with English abstract).

[11] Coello CAC. Treating constraints as objectives for single-objective evolutionary optimization. Engineering Optimization, 2000, 32(3):275-308.

[12] Coello CAC. Constraint-Handling using an evolutionary multiobjective optimization technique. Civil Engineering and Environmental Systems, 2000,17(4):319-346.

[13] Shang RH, Ma WP. Immune clonal MO algorithm for ZDT problems. In: Jiao LC, Wang LP, Gao XB, Liu J, Wu F, eds. Lecture Notes in Computer Science, Vol.4222. Berlin, Heidelberg, New York: Springer-Verlag, 2006. 100-109. 
[14] Liu F, Yang HC. A clone based multicast algorithm with adjustable parameter. Journal of Software, 2005,16(1):145-150 (in Chinese with English abstract). http://www.jos.org.cn/1000-9825/16/145.htm

[15] Shang RH, Ma WP, Zhang W. Immune clonal MO algorithm for 0/1 knapsack problems. In: Jiao LC, Wang LP, Gao XB, Liu J, Wu F, eds. Lecture Notes in Computer Science, Vol.4221. Berlin, Heidelberg, New York: Springer-Verlag, 2006. 870-878.

[16] Pan ZJ, Kang LS, Chen YP. Evolutionary Computation. Beijing: Tsinghua University Press, 1998 (in Chinese).

\section{附中文参考文献:}

[1] 焦李成,杜海峰,刘芳,公茂果.免疫优化计算、学习与识别.北京:科学出版社,2006.

[4] 周育人,李元香,王勇,康立山.Pareto 强度值进化算法求解约束优化问题.软件学报,2003,14(7):1243-1249. http://www.jos.org.cn/ 1000-9825/14/1243.htm

[10] 林丹,李敏强,寇纪松.基于遗传算法求解约束优化问题的一种算法.软件学报,2001,12(4):628-632.

[14] 刘芳,杨海潮.参数可调的克隆多播路由算法.软件学报,2005,16(1):145-150. http://www.jos.org.cn/1000-9825/16/145.htm

[16] 潘正君,康立山,陈毓屏.演化计算.北京:清华大学出版社,1998.

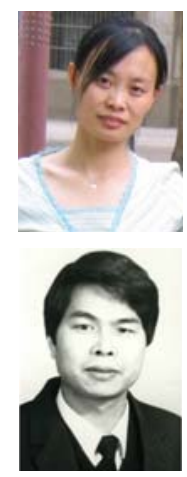

尚荣华 $(1979-)$,女,河北衡水人,博士,主 要研究领域为自然计算,工程优化,智能信 息处理.

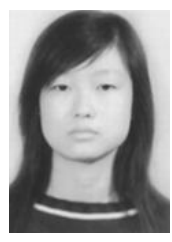

马文萍(1981－), 女, 博士, 讲师, 主要研究 领域为自然计算, 图像处理, 智能信息 处理.

焦李成(1959-), 男, 博士, 教授, 博士生导 师,CCF 高级会员, 主要研究领域为自然计 算,数据挖掘,图像处理,智能信息处理. 